\title{
DETERMINACIÓN DE ANTICUERPOS CONTRA EL VIRUS DE LA RETICULOENDOTELIOSIS AVIAR EN GALLINAS PONEDORAS MEDIANTE LA PRUEBA DE ELISA
}

\author{
Determination of Antibodies Against Reticuloendotheliosis Virus in LaYer \\ Hens BY The ELISA Test
}

\author{
Melina Grados T. ${ }^{1,2}$, Eliana Icochea D. ${ }^{1,3}$, Rosa Gonzáles V. ${ }^{1}$ y \\ Alberto Manchego S. ${ }^{4}$
}

\section{Resumien}

El objetivo del presente estudio fue determinar la presencia de anticuerpos contra el virus de la Reticuloendoteliosis (REV) en lotes de gallinas ponedoras utilizando la prueba de ELISA con kits comerciales. Se tomaron 630 muestras de suero, entre julio de 2004 a marzo de 2005, de 42 lotes en producción de 28 granjas comerciales del departamento de La Libertad y la provincia de Lima. Se encontró el 33.3\% (14/42) de lotes seropositivos, siendo $22.2 \%$ (6/27) en Lima y 53.33\% en La Libertad, sin encontrar asociación estadística de la infección con antecedentes de problemas tumorales, grupo etáreo y línea genética. Este es un primer estudio realizado en gallinas ponedoras comerciales cuyos resultados evidencian la presencia del REV en el Perú.

Palabras clave: Virus de la Reticuloendoteliosis, REV, anticuerpos, prueba de ELISA, gallinas ponedoras

\section{Abstract}

The objective of this study was to evaluate the presence of antibodies against Reticuloendotheliosis virus (REV) in flocks of layers hens using commercial ELISA kits. A total of 630 serum samples from 42 flocks in production of 28 commercial farms in the department of La Libertad and the province of Lima were collected from July 2004 till March 2005. The results indicated that $33.3 \%$ (14/42) flocks were seropositive [22.2\% $(6 / 27)$ in Lima and $53.3 \%(8 / 15)$ in La Libertad respectively], and without significant association between the infection and history of tumor problems, age, and genetic line. This is the first study conducted in commercial layer hens and the results showed the presence of the REV in Peru.

Key words: Reticuloendotheliosis virus, REV, antibodies, ELISA test, layers hens

\footnotetext{
${ }^{1}$ Laboratorio de Patología Aviar, ${ }^{4}$ Laboratorio de Microbiología y Parasitología Veterinaria, Facultad de Medicina Veterinaria, Universidad Nacional Mayor de San Marcos, Lima

${ }^{2}$ E-mail: melivet@gmail.com

${ }^{3}$ E-mail: eliana.icochea@gmail.com
} 


\section{INTRODUCCION}

Se denomina reticuloendoteliosis a un grupo de síndromes patológicos que se presentan en varias especies de aves, y es causado por la infección con los retrovirus del grupo de la Reticuloendoteliosis (REV). La enfermedad incluye: a) Síndrome del enanismo, b) Neoplasia crónica de los tejidos linfoides y otros, y c) Neoplasia aguda de las células reticulares (Salem et al., 1989; Payne, 1998; Witter y Fadly, 2003). Los REV son retrovirus no relacionados a los del grupo Leucosis/Sarcoma, y pertenecen al género de virus relacionados con la leucemia murina (Witter, 2000). Actualmente se consideran dentro de los virus tipo $\mathrm{C}$ de los mamíferos, del subgénero de los virus de la Reticuloendoteliosis (Ritchie et al., 1999).

El REV no está muy difundido, pero su presentación en varios países es mucho más amplia de lo que se creía inicialmente (Manual Merck, 2000). Se le encuentra en granjas de pollos y pavos de Israel, Australia, Japón y el sureste de EEUU (Jordan, 1990; Manual Merck, 2000). La variedad de hospederos es mucho mayor que para otros virus tumorales aviares (Witter, 1997a; Manual Merck, 2000). La incidencia de la enfermedad clínica asociada al REV varía de esporádica a insignificante; sin embargo, la prevalencia medida por frecuencia de lotes positivos es relativamente alta y tiende a incrementarse (Witter, 1997b).

La transmisión horizontal es probablemente más importante que la vertical (Manual Merck, 2000). Los porcentajes de transmisión vertical son generalmente más bajos para REV que para el virus de Leucosis Aviar (Witter, 1997b). La transmisión horizontal se presenta generalmente en aves jóvenes cuando el virus es difundido por las heces y fluidos corporales (Ritchie et al., 1999). Las fuentes de infección incluyen galpones contaminados, insectos y otros reservorios biológicos (Witter y Fadly, 2003). Asimismo, se ha reportado la contaminación accidental con REV de vacunas a virus vivos de Difteroviruela Aviar y Enfermedad de Marek (Davidson y Malkinson, 1997; Witter y Fadly, 2003), y de la vacuna contra el Herpesvirus en pavos (Medina y Ghazikhanian, 1997).

La Oficina Internacional de Epizootias (OIE) considera a la enfermedad neoplásica aviar producida por el REV, juntamente con los virus de la enfermedad de Marek y de la Leucosis Aviar, como importantes desde el punto de vista comercial, debido a las pérdidas económicas generadas por la mortalidad y el bajo rendimiento productivo que ocasionan en las aves (Payne y Venugopal, 2000). Asimismo, hay una creciente preocupación por este virus ante los decomisos de aves por la presentación de tumores. El virus de la REV, por ser un agente contaminante potencial de vacunas a virus vivo aviar, ha forzado a los productores de vacunas y a las empresas productoras de líneas genéticas libres de patógenos específicos (SPF) a mantener una vigilancia permanente de sus productos. Por otro lado, a la REV se le considera una barrera para la exportación de la progenie de lotes infectados, limitando el comercio internacional de aves y sus productos avícolas (Witter, 2000).

Actualmente, se encuentran disponibles kits comerciales de ELISA para la detección de anticuerpos en suero de pollos. Esta es una prueba inmunoenzimática eficaz, rápida, sensible y útil para las detección de anticuerpos en aves positivas, así como para confirmar la ausencia del virus en lotes libres de patógenos o lotes de reproductores para la exportación (Witter, 2000). En el Perú, en un estudio previo se demostró que todos los lotes muestreados de gallinas reproductoras eran negativos a la presencia de anticuerpos al REV (Salas, 2005); sin embargo, no hay datos en gallinas de postura comercial. Por tal motivo, el objetivo del presente estudio fue determinar la presencia de anticuerpos contra el virus de la Reticuloendoteliosis en lotes de gallinas ponedoras de la provincia de dos regiones del Perú mediante la prueba comercial de ELISA. 


\section{Materiales y Métodos}

El estudio se realizó en 28 granjas comerciales de la provincia de Lima y del departamento de La Libertad. El procesamiento de las muestras se llevó a cabo en el Laboratorio de Patología Aviar de la Facultad de Medicina Veterinaria de la Universidad Nacional Mayor de San Marcos, Lima.

El tamaño muestral $(n=37)$ se determinó considerando el número total de lotes en producción de granjas formales en la provincia de Lima y departamento de La Libertad ( $\mathrm{n}=187$ ) y un nivel de confianza del $95 \%$. Se fijó arbitrariamente una prevalencia límite de $7 \%$ debido a que no existen estudios previos en el país en lotes de gallinas ponedoras comerciales. Además, se consideró emplear un mínimo de 15 aves por lote (Witter, 1992).

En la práctica, se colectaron muestras de sangre de 630 aves, procedentes de 42 lotes de gallinas ponedoras en producción, durante los meses de julio de 2004 a marzo de 2005. Las aves se clasificaron de acuerdo a tres variables: antecedentes por problemas tumorales (presencia o ausencia), líneas genéticas (Hy Line, Isa Brown, Lohman y Brown Link), y grupos etáreos (20-35, 36-50, 51-65, >65 semanas de edad).

El muestreo se realizó al azar y las muestras de sangre se obtuvieron por punción de la vena braquial $(2 \mathrm{ml})$. El suero resultante fue conservado en congelación a $-20{ }^{\circ} \mathrm{C}$ hasta la realización de la prueba diagnóstica de ELISA utilizando kit comerciales (IDEXX Laboratories), para la determinación de anticuerpos contra el virus de Reticuloendoteliosis Aviar. Las muestras fueron diluidas 1:500 en solución buffer siguiendo el procedimiento indicado por el laboratorio fabricante. Las densidades ópticas fueron medidas a $650 \mathrm{~nm}$ por un lector de ELISA ELX 800 (Biotek Intruments, Inc.). Las muestras de suero que tuvieron cocientes M/P $£ 0.05$ se consideraron negativas y aquellas con cocientes $>0.5$ se consideraron positivas.
Los resultados generales se expresaron en porcentaje con su intervalo de confianza al $95 \%$. Un lote fue considerado positivo si al menos un suero fue positivo a la prueba. Se evaluó la asociación estadística entre las variables antecedentes de problemas tumorales, grupo etáreo, y líneas genéticas con el resultado de la prueba diagnóstica, a través de la prueba de Chi Cuadrado.

\section{Resultados}

De los 42 lotes en producción de gallinas ponedoras en estudio, 14 dieron positivo a la presencia de anticuerpos contra la REV, lo que significa una frecuencia general de $33.3 \pm 14.2 \%$; siendo mayor al $7 \%$ de la prevalencia referencial esperada. No se observó diferencias estadísticas entre la presencia de anticuerpos contra REV y la historia de antecedentes tumorales, grupo etáreo o línea genética (Cuadro 1). Cinco lotes (36\%) tuvieron menos del $25 \%$ de sueros serorreactores y nueve lotes (64\%) presentaron $25 \%$ ó más de sueros reactores.

\section{Discusión}

La seropositividad en 14 de 42 lotes evaluados $(33.3 \%)$ representa un alto porcentaje de la presencia del virus en gallinas ponedoras en el país. Las dos granjas comerciales que participaron en el estudio están ubicadas en zonas de alta densidad poblacional de aves y se caracterizan por tener la mayor población de gallinas ponedoras en el país. Los resultados de la prueba de ELISA se interpretaron como positivo si existía al menos un suero positivo dentro del grupo de aves, y en el presente estudio se encontró gran cantidad de aves serorreactoras dentro de cada lote y con valores de densidades ópticas mucho mayores que aquellos de los controles positivos. En el estudio de Salas (2005) en lotes de gallinas reproductoras de postura y carne mayores 
Cuadro 1. Frecuencia de lotes de gallinas ponedoras comerciales seroreactoras a reticuloentolesiosis (REV), según su procedencia, historia de problemas tumorales, grupo etáreo, y líneas genéticas (2004-2005)

\begin{tabular}{|c|c|c|}
\hline & \multicolumn{2}{|c|}{ Lotes } \\
\hline & $\begin{array}{l}\mathrm{N}^{\circ} \text { de muestras positivas / } \\
\mathrm{N}^{\circ} \text { muestras examinadas }\end{array}$ & $\begin{array}{c}\text { Frecuencia } \\
(\%)\end{array}$ \\
\hline \multicolumn{3}{|l|}{ Procedencia } \\
\hline Lima & $6 / 27$ & $22.2^{\mathrm{a}}$ \\
\hline La Libertad & $8 / 15$ & $53.3^{\mathrm{b}}$ \\
\hline \multicolumn{3}{|l|}{ Problemas tumorales } \\
\hline Positivo & $5 / 13$ & 38.5 \\
\hline Negativo & $9 / 29$ & 31.0 \\
\hline \multicolumn{3}{|c|}{ Grupo etáreo (semanas de edad) } \\
\hline 20 a 35 & $4 / 8$ & 50.0 \\
\hline 36 a 50 & $4 / 10$ & 40.0 \\
\hline 51 a 65 & $2 / 16$ & 12.5 \\
\hline$>65$ & $4 / 8$ & 50.0 \\
\hline \multicolumn{3}{|l|}{ Líneas genéticas } \\
\hline Hy Line & $10 / 22$ & 45.5 \\
\hline Isa Brown & $2 / 7$ & 28.6 \\
\hline Lohman & $1 / 11$ & 9.1 \\
\hline Brown Link & $1 / 2$ & 50.0 \\
\hline Total & $14 / 42$ & $33.3 \pm 14.2^{1}$ \\
\hline
\end{tabular}

1 Intervalo de confianza del 95\%

${ }^{a, b}$ Superíndices diferentes indican diferencias estadísticas dentro de cada variable $(p<0.05)$

de 50 semanas de edad solo se detectó tres sueros positivos de un total de 180 analizados, y los valores de densidad óptica fueron inferiores a los controles positivos.

Los resultados del estudio hecho por Salas (2005) minimizan la posibilidad de infección por transmisión vertical al nacimiento en pollitas ponedoras comerciales. No obstante, se debe tener en cuenta las deficientes medidas de bioseguridad que existen en la crianza de aves de postura comercial comparadas con el alto nivel existente en granjas de reproductoras de líneas pesadas. Esta situación conlleva a un alto riesgo de contaminación horizontal durante la crianza en gallinas de postura, haciendo posible los hallazgos de seroconversión detectados en el presente estudio. 
La frecuencia de lotes seropositivos al REV es comparable al reporte de Witter et al. (1982) en EEUU donde se encontró $21 \%$ de infección en 101 lotes de ponedoras usando la prueba de inmunofluorescencia indirecta; sin embargo, en la zona se tenía la historia de la presencia del REV, mas no así en el Perú donde no se tiene antecedentes de este tipo de problemas tumorales. Por otro lado, en otros estudios se ha documentado la detección de anticuerpos contra REV en 60$80 \%$ de las aves en algunas parvadas de EEUU y Japón, indicando que la frecuencia de animales seropositivos está aumentando (Witter, 2000).

La neoplasia linfoide crónica y el síndrome del enanismo son las manifestaciones clínicas más comunes que ocasiona la infección por el REV (Whiteman y Bickford, 1996), aunque también puede producir tumores similares a los de la Enfermedad de Marek y a los de Leucosis Aviar. En el presente estudio no fue posible establecer una relación significativa entre lotes con antecedentes tumorales y la infección con el REV, pero tampoco se descarta que los problemas tumorales hayan podido ser causados por la REV.

Actualmente, las manifestaciones clínicas en pollos se asocian principalmente al uso de vacunas contaminadas con REV (Witter, 1997a). Se han encontrado tumores linfoides crónicos en Australia en un lote de ponedoras luego de la administración de una vacuna contra la enfermedad de Marek, contaminada con REV (Witter, 2000); y en EEUU se observaron linfomas relacionados con REV en dos parvadas de reproductoras de carne por el uso de una vacuna de viruela aviar contaminada con este agente (Witter, 2000). No se conoce la fuente de infección en el país, aunque es muy probable que la vía sea la horizontal; no obstante, no debe descartarse la posibilidad de vacunas contaminadas con REV como fuente de infección, especialmente en los lotes con antecedentes tumorales. Así mismo, existe la posibilidad de reservorios naturales del virus como fuentes de infección (Witter, 1997b), entre ellos las aves silvestres (Zavala, 2004). Además, se debe considerar la posible transmisión por mosquitos (Witter,1997b).

La edad de las aves es un factor a considerar en la interpretación de los resultados serológicos. Los títulos de anticuerpos podrían ser bajos si la infección ocurre a edades tempranas, de allí que sería necesario hacer una segunda evaluación un mes después. Por otro lado, los títulos de anticuerpos declinan más rápidamente en aves de edades mayores $(\mathrm{R}$. Witter, comunicación personal). Sin embargo, en este estudio no se encontró una asociación significativa por efecto de la edad.

La línea genética fue una variable en estudio debido a que el virus se transmite verticalmente; sin embargo, las cuatro líneas de aves evaluadas estuvieron afectadas sin encontrarse diferencia estadística entre ellas.

En el $64 \%$ de los lotes de postura que resultaron positivos a la prueba de ELISA, por lo menos el $25 \%$ de las aves positivas fue positivo a REV y con altos valores de seroconversión. Se asevera que títulos altos de anticuerpos aunados con un moderado porcentaje de sueros positivos en los lotes permite confirmar la presencia del virus en dichos lotes (R. Witter, comunicación personal). Por otro lado, el control sanitario de ingreso de lotes de pollos importados en Israel considera como positivo a REV a un lote si el $50 \%$ o más de los sueros son positivos a la prueba de ELISA Indirecta, aunque recomiendan un monitoreo adecuado de las aves mediante esta prueba y el PCR (Davidson y Malkinson, 1997).

Debido a la evidencia del agente infeccioso en el país y la creciente prevalencia en otros países, se debe considerar la vigilancia epidemiológica del REV. 


\section{Conclusiones}

? Se encontró serorreacción al virus de la Reticuloendoteliosis mediante la prueba de ELISA, en el 33.3\% de 42 lotes de aves de postura comercial estudiados.

? No se encontró asociación estadística entre lotes positivos y antecedentes tumorales, grupo etáreo y línea genética.

\section{Literatura Citada}

1. Davidson I, Malkinson M. 1997. Epidemiology and control of Reticuloendotheliosis Virus in chickens and turkeys in Israel. En: Avian Tumor Viruses Symposium. 40 ${ }^{\text {th }}$ Annual Meeting AAAP. Reno, Nevada. p 70-75.

2. Jordan F. 1990. Reticuloendotheliosis. In: Poultry Diseases. $3^{\text {rd }}$ ed. Liverpool: Ed. Baillere Tindall. p 118-120.

3. Medina A, Ghazikhanian Y. 1997. Reticuloendotheliosis virus infection in turkeys: Industry perspective. In: Avian Tumor Viruses Symposium. 40 $0^{\text {th }}$ Annual Meeting AAAP. Reno, Nevada. p 67-68.

4. Manual Merck de Veterinaria. 2000. $5^{\text {ta }}$ ed. Barcelona, España: Ed. Oceano/ Centrum. $2558 \mathrm{p}$.

5. Payne L. 1998. Retrovirus-Induced diseases in poultry. Poultry Sci 77: 12041210.

6. Payne L, Venugopal K. 2000. Enfermedades neoplásicas: enfermedad de Marek, leucosis aviar y reticuloendoteliosis aviar. Rev Sci Tech OIE 19(2): 544-564. [Internet] Disponible en: http://www.oie.int/esp/publicat/rt/ 1902/e r19213.htm

7. Ritchie B, Branson W, Harrison $G$. 1999. Retroviridae. In: Avian medicine: principles and application. Ch. 32. Florida, USA: HBD Internacional. p 931-936.

8. Salas E. 2005. Evidencia serológica contra el virus de Reticuloendoteliosis
Aviar en gallinas reproductoras mayores de 50 semanas de edad mediante la prueba de ELISA. Tesis de Médico Veterinario. Lima: Facultad de Medicina Veterinaria, Univ. Nacional Mayor de San Marcos. 50 p.

9. Salem M, Eckroade J, Keller L. 1989. Experiences using ELISA in a Reticuloendotheliosis outbreak in turkeys. In: Proc XXXVIII Western Poultry Disease Conference. Arizona. p 174175.

10. Whiteman C, Bickford S. 1996. Avian diseases manual. $4^{\mathrm{h}}$ ed. Pensylvannia: American Association of Avian Pathologists.

11. Witter R. 1992. Reticuloendotheliosis. In: A laboratory manual for the isolation and identification of avian pathogens. $3^{\text {rd }}$ ed. Florida, USA: Kendall/Hunt Publishing Co. p 143-148.

12. Witter R. 1997a. Reticuloendotheliosis virus: An overview of current issues. In: Avian Tumor Viruses Symposium. 40 Annual Meeting AAAP. Reno, Nevada. p 63-64.

13. Witter R. 1997b. Avian tumor viruses: Persistent evolving pathogens. Acta Vet Hung 45(3): 251-266.

14. Witter R. 2000. Reticuloendotheliosis. In: Calnek BW, Barnes Saif HJ, eds. Diseases of poultry. $2^{\text {nd }}$ ed. Cap.17. Iowa State University Press. p 478-496.

15. Witter $R$, Fadly A. 2003. Reticuloendotheliosis. In: Diseases of poultry. $11^{\text {th }}$ ed. Cap.15. Iowa State University Press. p 517-529.

16. Witter R, Peterson I, Smith E, Johnson D. 1982. Serologic evidence in commercial chicken and turkey flocks of infection with Reticuloendotheliosis virus. Avian Dis 26: 753-762.

17. Zavala G. 2004. Enfermedades tumorales en aves. Curso Segunda Especialización en Aves. Lima: Facultad de Medicina Veterinaria, Univ. Nacional Mayor de San Marcos. 